Second United Nations

International Conference

on the Peaceful Uses

of Atomic Energy
$\mathrm{A} / \mathrm{CONF} .15 / \mathrm{P} / 629$

Abstract (USA)

7 March 1958

ORIGINALE ENGIISH

Confidential until official release during Conference

A SURVEY OF NUCLEAR REACTOR KINETICS

Prepared By

E. R. Cohen, D. I. Hetrick and W. A. Horning

Abstract

The Boltzmann equation for the neutron distribution in a reactor is first reduced to the energy independent, space dependent equation in terms of neutron importance functions. It is therefore possible to obtain equivalent "one-group" neutron models which are more general than the simple "thermal group" approximation. The one group space dependent equation can then be used to investigate the effects of spatially localized perturbations. In particular, the "rod-drop" method of determining control rod worth is discussed in general terms as an example of such spatially localized perturbations. The conditions for stability of a reactor against flux oscillations initiated by spatially localized perturbations are also investigated.

The equations are then reduced to space and energy independent form and several cases are presented for which analytic solutions are possible. In general, however, the feedback of the power and temperature variables alter the reactivity and introduce non-linear terms into the equations and no analytic solutions exist. In such cases numericol methods must be used. Several methods present themselves. There are various possible nurnerical procedures based upon the replacement of the differential equations by difference equations and the advantages and disadvantages of these are reviewed. An alternative approach which can be quite useful takes as 1ts starting point the formulation of the system as a set of coupled integral equations and the numerical replacement of integrals by approximating sums. In all of these methods the problems of convergence and numerical stability are important, and the methods for formulating convergence and stability criteria are discussed.

In reactor transients in which the reactor is self-limiting, the nature of the feedback mechanism is of utmost importance. The types of feedback mechanisms which are present in homogeneous solution reactors (KEWB) and in plate-type reactors (SPERT, OMRE, EBWR) are discussed and certain general stability criteria are evaluated. The general results are illustrated with data on the performance of the SPERT and IEWB tests.

In the aqueous homogeneous reactor, two reactivity feedback mechanisms may be distinguished: a prompt negative temperature coefficient arising primarily from thermal expansion of the fuel solution, and a delayed negative void coefficient resulting from the formation of bubbles of radiolytic decomposition gas. Data from the Kinetic Experiment Water Boiler are present, together with a discussion of various mathematical models which have been used to simulate the dynamic behavior of this reactor. 


\section{DISCLAIMER}

This report was prepared as an account of work sponsored by an agency of the United States Government. Neither the United States Government nor any agency Thereof, nor any of their employees, makes any warranty, express or implied, or assumes any legal liability or responsibility for the accuracy, completeness, or usefulness of any information, apparatus, product, or process disclosed, or represents that its use would not infringe privately owned rights. Reference herein to any specific commercial product, process, or service by trade name, trademark, manufacturer, or otherwise does not necessarily constitute or imply its endorsement, recommendation, or favoring by the United States Government or any agency thereof. The views and opinions of authors expressed herein do not necessarily state or reflect those of the United States Government or any agency thereof. 


\section{DISCLAIMER}

Portions of this document may be illegible in electronic image products. Images are produced from the best available original document. 
The interpretation of spontaneous power ascillations in SPERT I has depended on two time delays: 1) That for super heat in the water. to form into steam voids; 2) The residence time of steam voids in the core. It has been possible to choose these two delays to account for the principal observed features of resonance instability in SPERT I, namely: 1) The dependence of frequency on critical power; 2) The dependence of power peak width on amplitude; 3) The relation between power oscillations and the accompanying fuel plate temperature oscillations. The above theory will be confirmed if out-of-pile measurements of the above two delay times yield the same values as those now required to fit SPERT data. 


\title{
SOME TOPICS IN REACTOR KINETICS
}

by

\author{
E. Richard Cohen*
}

\section{FORMULATION OF THE KINETIC BEHAVIOR OF A REACTOR}

The diffusion of neutrons is determined by the Boltzmann equation which is a detailed statement of the conservation of neutrons in every infinitesimal element of phase space. In general this is a partial differential -quation in seven independent variables and explicit solutions are available in only a discouragingly few cases. Most often the symmetry of the problem or a specialization of the system will reduce the number of variables. Even so, approximate solutions are usually the only ones found. In reactor kinetic studies it is common to integrate out the spatial and energy variables so that time is the only independent variable. It is loosely states that the solution then gives a description of the time dem pendence of the total neutron population in the reactor. There are, however, several applications where this elementary description is inadequate and can lead to erroneous results. This section shall investigate the validity and the basic formulation of the space-energy independent equations.

We write the Boltzmann equation in the form

$$
\frac{\partial N}{\partial t}+\vec{v} \cdot \nabla N+\sigma v N=(T N)+(1-\beta) f(v)(F N)+\sum_{i} \lambda_{i} c_{i}(\vec{r}, t) f_{i}(v)+S(\vec{r}, t)
$$

where $N=N(\vec{r}, \vec{v}, t)$ is the neutron density in phase space at the point $\vec{r}, \vec{v}$. Because the neutron interactions depend only on the magnitude of $v$ and not on its orientation, it is often useful to write $v$ in polar coordinates $(v, \vec{\Omega})$ and to express the speed, $v$, in terms of either the energy or the lethargy. $\sigma$ is the total collision cross section, and

*Atomics International

A Division of North American Aviation, Inc.

P. 0. Box 309

Canoga Park, California

Contributors: D. L. Hetrick, Atomics International

W. A. Horning, Atomics International

A. F. Henry, Westinghouse Electric Corp. 
$v \sigma$ is the collision probability per neutron per unit time.

$$
\begin{aligned}
& (T N) \equiv \int v^{\prime} N\left(\vec{r}, \vec{v}^{\prime}, t\right) \sigma_{\text {in }}\left(\vec{v}^{\prime} \rightarrow \vec{v} ; \vec{r}\right) d \vec{v}^{\prime} \\
& (F N) \equiv \int v^{\prime} N\left(\vec{r}, \vec{v}^{\prime}, t\right) v \sigma_{f}\left(\vec{r}, \vec{v}^{\prime}\right) d \vec{v}^{\prime}
\end{aligned}
$$

$c_{i}(r)$ is the concentration of the $i$ th group of delayed neutron precursors, $\lambda_{i}$ is the precursor decay constant.

$f(v), f_{i}(v)$ are the velocity distributions of emitted neutrons from fission or from delayed neutron emission of group $i$.

The precursors satisfy the equations (in a fixed fuel reactor),

$$
\frac{d c_{i}}{d t}=\lambda_{i} c_{i}(\vec{r}, t)+\beta_{i}(F N)
$$

A certain amount of insight into the time dependent behavior of neutrons can be obtained from an expansion of $N(\vec{r}, \vec{v}, t)$ in terms of eigenfunctions. There are various ways in which such an expansion may be defined. Expansion in terms of spatial eigenfunctions are possible but these are primarily useful only for bare reactors. In general, spatial eigenvalue expansions can be considered as equivalent to a laplace transformation of the spatial coordinates. It is most common to expand either in terms of reactivity eigenfunctions or in terms of period eigenfunctions. These two basis systems are not identical. Reactivity eigenfunctions are defined by the equation

$$
\begin{aligned}
\vec{v} \cdot \nabla \varphi_{s}(\vec{r}, \vec{v}) & +\sigma(\vec{r}, \vec{v}) v \varphi_{s}(\vec{r}, \vec{v})-\left(T \varphi_{s}\right)=\frac{1}{k_{s}} \bar{f}(v)\left(F \varphi_{s}\right) \\
\bar{f}(v) & =(1-\beta) f(v)+\sum_{i} \beta_{i} f_{i}(v)
\end{aligned}
$$

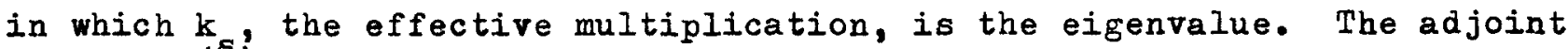
equation ( $\underline{\underline{S}})$ is*

$$
\begin{aligned}
& -\vec{v} \cdot \nabla \tilde{\varphi}_{s}+\sigma(\vec{r}, \vec{v}) v \tilde{\varphi}_{s}-\left(T^{+} \tilde{\varphi}_{s}\right)=\frac{1}{k_{s}} \nu \sigma_{f}(\vec{r}, \vec{v}) v\left(\bar{f} \tilde{\varphi}_{s}\right) \\
& \left(T^{+} \tilde{\varphi}_{s}\right) \equiv v \int \sigma_{i n}\left(\vec{v} \rightarrow \vec{v}_{j}^{\prime} \vec{r}\right) \tilde{\varphi}_{s}\left(\vec{r}, \vec{v}^{\prime}\right) d \vec{v}^{\prime} \\
& \left(\bar{f} \tilde{\varphi}_{s}\right) \equiv \int f\left(v^{\prime}\right) \tilde{\varphi}_{s}\left(\vec{r}, \vec{v}^{\prime}\right) d \vec{v}^{\prime}
\end{aligned}
$$

* As usually formulated, a factor of $v$ is cancelled out of Eq (4). From the standpoint of mathematical symmetry it is more convenient to retain this factor. In this we follow Ussachoff (I), but see, for example, Henry (ㄹ). 
The orthogonality relation for these functions is

$$
\left(\frac{1}{k_{\sigma}}-\frac{1}{k_{s}}\right) \int\left(\bar{f} \tilde{\varphi}_{\sigma}\right)\left(\nu \sigma_{f} \vee \varphi_{s}\right) d \vec{r}=0
$$

On the other hand, the period eigenfunction are defined by

$$
\begin{aligned}
& -\vec{v} \cdot \nabla \psi_{s}(\vec{r}, \vec{v})-\sigma(\vec{r}, \vec{v}) v \psi_{s}(\vec{r}, \vec{v})+\left(T \psi_{s}\right) \\
& +(1-\beta) f(v)\left(F \psi_{s}\right)+\sum_{i} \lambda_{i} f_{i}(v) \xi_{i s}(\vec{r})=\omega_{s} \psi_{s}(\vec{r}, \vec{r}) \\
& \beta_{i}\left(F \psi_{s}\right)-\lambda_{i} \xi_{i s}(\vec{r})=\omega_{s} \xi_{i s}(\vec{r})
\end{aligned}
$$

in which the eigenvalue is $\omega_{\mathbf{s}}$.

The adjoint of $\mathrm{Eq}(6)$ is

$$
\begin{gathered}
\vec{v} \cdot \nabla \tilde{\psi}_{\sigma}-\sigma(\vec{r}, \vec{r}) v \tilde{\psi}_{\sigma}+\left(T^{+} \tilde{\psi}_{\sigma}\right)+\nu \sigma_{f} v\left[(1-\beta)\left(f \tilde{\psi}_{\sigma}\right)+\sum_{i} \beta_{i} \tilde{\xi}_{i \sigma}\right]=\omega_{\sigma} \tilde{\psi}_{\sigma} \\
\lambda_{i}\left[\left(f_{i} \tilde{\psi}_{\sigma}\right)-\tilde{\xi}_{i \sigma}\right]=\omega_{\sigma} \tilde{\xi}_{i \sigma}
\end{gathered}
$$

The delayed neutron precursors can be considered as the components of the neutron density in an extended velocity space. This extended space consists of the physical velocity space plus a set of discrete points at which there are delta function distributions of neutrons. The inclusion of the delayed precursors therefore adds a summation over a discrete set to the integration over velocities. The orthogonality relationship is given by

$$
\begin{aligned}
& \int\left[\left(\tilde{\psi}_{\sigma} \psi_{s}\right)+\sum_{i} \tilde{\xi}_{i \sigma} \xi_{i s}\right] d \vec{r}=\delta_{\sigma s} \\
& =\int\left[\left(\tilde{\psi}_{\sigma} \psi_{s}\right)+\sum_{i} \frac{\beta_{i} \lambda_{i}\left(f_{i} \tilde{\psi}_{\sigma}\right)\left(F \psi_{s}\right)}{\left(\lambda_{i}+\omega_{\sigma}\right)\left(\lambda_{i}+\omega_{s}\right)}\right] d \vec{r} .
\end{aligned}
$$

The precursor component may be eliminated from Eq (6) but only at the expense of losing the simple eigenvalue character. The two equations of (6) can be replaced by the single equation

$$
\begin{aligned}
-\vec{v} \cdot \nabla \psi_{s}(\vec{r}, \vec{v})-\sigma(\vec{r}, \vec{v}) v \psi_{s}(\vec{r}, \vec{v}) & +\left(T \psi_{s}\right)+\bar{f}(v)\left(F \psi_{s}\right) \\
= & \omega_{s}\left\{\psi_{s}+\sum_{i} \frac{\beta_{i} f_{i}(v)\left(F \psi_{s}\right)}{\lambda_{i}+\omega_{s}}\right\} \\
& -3-
\end{aligned}
$$


There will now be a set of values $\omega_{s}$ for each eigenfunction $\psi_{s}$.

If we make an expansion of $N(\vec{r}, \vec{v}, t)$ in terms of the eigenfunction set $\psi_{s^{\prime}} N(\vec{r}, \vec{v}, t)=\sum_{s} A_{s}(t) \psi_{s}(\vec{r}, \vec{v})$, Eqs (1) and (2) yield the equations for the time behavior of the 总odal amplitudes

$$
\frac{d A_{s}}{d t}-\omega_{s} A_{s}(t)=S_{s}(t)=\iint \psi_{s}(\vec{r}, \vec{v}) S(\vec{r}, \vec{v}, t) d \vec{v} d \vec{r}
$$

From this equation we may easily obtain the usual expression for the physical interpretation of the adjoint function. Let the source be given by $S(\vec{r}, \vec{v}, t)=\delta(\vec{r}-\vec{r}) \delta(\vec{v}-\vec{v}) \delta(t) ; i . e_{0}$, at time $t=0$ a pulse of neutrons is injected ${ }^{\circ}$ into the system at the point $\vec{r}_{0}$ with velocity $\vec{v}_{0}{ }^{\circ}$ The amplitude of the $\sigma$ mode is then

$$
A_{\sigma}(t)=\tilde{\psi}_{\sigma}\left(\vec{r}_{0}, \vec{v}_{0}\right) e^{\omega_{\sigma} t}
$$

and hence the adjoint function is the amplitude of the neutron density resulting from a pulse neutrons injected into the reactor at a given point with given velocity. If the reactor is subcritical ( $\omega_{\sigma}<0$ for all $\sigma$ ), a constant source of So neutrons per second will be multiplied by the system and the equilibrium neutron amplitudes of each mode will be

$$
A_{\sigma}=\frac{S_{0}}{-\omega_{\sigma}} \tilde{\psi}_{\sigma}\left(\vec{r}_{0}, \vec{v}_{0}\right)
$$

The alternate expansion, in terms of the reactivity eigenfunctions $\varphi_{s}$ yields

$$
\begin{gathered}
N(\vec{r}, \vec{v}, t)=\sum B_{s}(t) \varphi_{s}(\vec{r}, \vec{v}) \\
\sum_{s}\left[l_{\sigma s} \frac{d B_{s}}{d t}-B_{s}\left(\rho_{\sigma} \delta_{\sigma s}-\beta_{\sigma s}\right)\right]=\sum \lambda_{i} D_{i \sigma}+S_{\sigma}^{*} \\
\frac{d D_{i \sigma}+\lambda_{i} D_{i \sigma}=\sum_{s} \beta_{\sigma s}^{i} B_{s}}{d t}
\end{gathered}
$$

where $l_{\sigma s}=\int\left(\tilde{\varphi}_{\sigma} \varphi_{s}\right) d \vec{r}$

$$
\begin{aligned}
& \rho_{\sigma}=\left(k_{\sigma}-1\right) / k_{\sigma} \\
& \beta_{\sigma s}^{i}=\beta_{i} \int\left(f_{i} \tilde{\varphi}_{\sigma}\right)\left(\nu \sigma_{f} v \varphi_{s}\right) d \vec{r} ; \quad \beta_{\sigma s}=\sum_{i} \beta_{\sigma s}^{i} \\
& D_{i \sigma}=\int\left(f_{i} \tilde{\varphi}_{\sigma}\right) c_{i}(\vec{r}, t) d \vec{r} ; \quad S_{\sigma}^{*}=\int\left(\tilde{\varphi}_{\sigma} S\right) d \vec{r}
\end{aligned}
$$

In all of these integrals the normalization used is 


$$
\int\left(\bar{f} \tilde{\varphi}_{\sigma}\right)\left(\nu \sigma_{f} \vee \varphi_{s}\right) d \vec{r}=\delta_{\sigma s}
$$

where, as previously defined, the parenthesis indicates integration over all velocities.

From equations (10.1) and (10.2) we obtain a generalized inhour equation. In place of the usual polynomial in $p$ (the temporal laplace transform variable) the generalized equation is of infinite degree and is represented as the vanishing of an infinite determinant,

$$
\left|\left\{l_{\sigma s}+\sum_{i} \begin{array}{c}
\beta_{\sigma s}^{i} \\
\lambda_{i}+p
\end{array}\right\} p-\rho_{\sigma} \delta_{\sigma s}\right|=0
$$

The same result may be obtained by expanding the eigenfunction $\psi_{s}(\vec{r}, \vec{v})$ as a series in the independent set $\varphi_{\mathbf{s}}(\vec{r}, \vec{v})$.

If the physical parameters of the system are time-dependent there are two possible approaches. The basis system for the eigenfunction expansion can be based on a time independent reactor configuration corresponding to some standard conditions and reactivity changes in the reactor can be defined relative to this standard configuration. It is also possible in principle to define a time dependent basis system and in this case the eigenfunctions and the adjoint functions must be determined as a function of time.

If we choose to keep a fixed eigenvector basis it is most convenient to use the reactivity eigenvector system; the only change in equation ( 10.1 ) is then that the term $\rho_{\sigma} \delta_{\sigma s}$ is replaced by $\rho_{\sigma} \delta_{\sigma s}+R \sigma_{s}(t)$. The reactivity matrix $R_{\sigma}(t)$ is the generalization of the reactivity change between the standard reactor configuration and the instantaneous reactor configuration:

$$
R_{\sigma_{s}}(t)=-\int\left(\tilde{\varphi}_{\sigma} \vee \delta \sigma \varphi_{s}\right) d \vec{r}+\int\left(\tilde{\varphi}_{\sigma}\left(\delta T \varphi_{s}\right)\right) d \vec{r}+\int\left(\tilde{\varphi}_{\sigma} \delta \bar{f}\left(F \varphi_{s}\right)\right) d \vec{r}
$$

where the operator $\delta$ indicates the change in the physical parameter from its standard value. The solutions of the infinite set of equations Eq $(10.1),(10.2),(13)$, is formidable and in general it is feasible only to calculate the fundamental eigenfunction and eigenvalues. Hence this formulation is not of particular utility for practical calculation although it helps to clarify the approximations which are inherent in the usual procedures. 
Because of the difficulties inherent in an expansion of the neutron density in terms of eigenfunctions (not the least of these being the question of completeness of such an expansion), A. F. Henry (ㄹ) has investigated the utility of a formulation which avoids a direct identification with eigenvalues. In essence, Henry's formulation uses only the fundamental mode of the adjoint solution, $\mathrm{Eq}$ (4); the expansion of $N$ in terms of eigenfunctions, however, is not made and $\mathrm{Eq}$ (10.I) is therefore replaced by an equation which is formally equivalent to the usual one group space independent model. If we write $N(\vec{r}, \vec{v}, t)=$ $N_{0}(\vec{r}, \vec{v}, t) A(t)$ with

$$
\int\left(\bar{f}_{0} \tilde{\varphi}_{0}\right)\left(F N_{0}\right) d \vec{r}=1
$$

we obtain

$$
\begin{aligned}
l \frac{d A}{d t} & =(\rho-\bar{\beta}) A(t)+\sum \lambda_{i} D_{i}(t)+S^{*}(t) \\
\frac{d D_{i}(t)}{d t} & =-\lambda_{i} D_{i}(t)+\beta_{e f f}^{i} A(t) \\
l & \equiv \int\left(\tilde{\varphi}_{0} N_{0}\right) d \vec{r} \\
\rho & \equiv \rho_{0}-\int\left(\tilde{\varphi}_{0} v \delta \sigma N_{0}\right) d \vec{r}+\int\left(\tilde{\varphi}_{0}\left(\delta T N_{0}\right)\right) d \vec{r} \\
& \quad+\int\left(\tilde{\varphi}_{0} \delta \bar{f}\left(F N_{0}\right)\right) d \vec{r} \\
\beta_{e f f}^{i} & \equiv \beta_{i} \int\left(f_{i} \bar{\varphi}_{0}\right)\left(F N_{0}\right) d \vec{r} \quad \bar{\beta} \equiv \sum_{i} \beta_{\text {eff }}^{i} \\
D_{i}(t) & \equiv \int\left(f_{i} \tilde{\varphi}_{0}\right) c_{i}(\vec{r}, t) d \vec{r} \quad S^{*}(t)=\int\left(\tilde{\varphi}_{0} S\right) d \vec{r}
\end{aligned}
$$

The error inherent in this formulation can be expressed in several ways. On the basis of the comparison between the eigenvalue expansion and the definitions of Eqs (15.1)-(15.4) we can say that Henry's formulation includes the higher modes of the neutron density but assigns to all of these neutrons the importance of neutrons in a fundamental mode. This error is most important in problems involving localized sources (or localized sinks).

Henry has emphasized the difficulties involved in the interpretation of experiments which involve spatially localized perturbations in terms of the space-independent equations. This reduction to spatial independence can be quite serious in the calibration of control rods by the so-called "rod drop" method. A given "shut down" (the negative of the reactivity of the system with rods inserted) can be achieved by infinitely many different control rod configurations. Each of these correspond to a 
different pattern of normal modes and hence the total neutron density in the system resulting from delayed neutron emission will change as the delayed neutron precursor distribution alters from its pre-shutdown to its post-shutdown shape. The reactivity of the system would then appear to change and in fact to increase with no changes in reactor parameters as the precursor source distribution readjusts itself to its equilibrium configuration.

In general an eigenfunction expansion, were it feasible, would yield an exact solution to the equations of reactor kinetics. However, the use of an adjoint function can reduce the problem of the time dependent Boltzmann equation to an approximation in which a sequence of time independent transport equations calculate the shape of the neutron density and the one group space independent kinetic equations determine the amplitude of this distribution.

\section{ANALYTIC SOLUTIONS OF THE KINETIC EQUATIONS}

The equations of reactor kinetics as usually formulated are given in Equations (14.1), (14.2). In addition, the reactivity $\rho$ must be specified in some way. Equation (15.2) provides one such specification in detail. If only the total power level of the reactor is of interest the reactivity is given as a function of time and of other integral quantities (average reactor fuel temperature, moderator temperature, etco) A quite different approach than the one taken here to the solution of the kinetics equations has been presented by Welton (3). The present survey is intended to complement Welton's discussion and to present topics which are not treated there. The reader is therefore directed to Welton's paper and to the references there quoted for another aspect of the general problem. It is certainly true that important topics still exist which are treated neither in that paper nor in this one.

When the reactivity, $\rho$, of Equation ( 14.1 ) is a constant, the solution of Equations (14.1), (14.2) are elementary and is easily calculated by the use of laplace transform theory (4). The neutron amplitude is given by

$$
n(t)=A(t)=\sum_{j} a\left(p_{j}\right) e^{p_{j} t} \quad c_{i}(t)=\frac{1}{l} D_{i}(t)=\sum_{j} \frac{\lambda_{i} a\left(p_{j}\right)}{\lambda_{i}+p_{j}} e^{p_{j} t}
$$

where $\mathrm{p}_{\mathrm{j}}$ are the roots of

$$
\rho=p\left\{l+\sum_{i} \frac{\beta_{\text {eff }}^{i}}{\lambda_{i}+p}\right\}
$$

and

$$
a(p)=\frac{n(0)+\sum_{i} \frac{\lambda_{i} c_{i}(0)}{\lambda_{i}+p}}{1+\frac{1}{\ell} \sum_{i} \frac{\beta_{\text {eff }}^{i} \lambda_{i}}{\left(\lambda_{i}+p\right)^{2}}}
$$


If the reactivity, $\rho$, is not a constant, analytic solutions become cumbersome and two alternatives are possible. We may either simplify the equations so that an analytic solution is feasible or we may handle the correct equations numerically. We shall discuss the numerical solutions below (Section III). For qualitative results it is often feasible to consider only one or two delayed neutron groups ( 5 ). In some important applications it is also possible to assume that the prompt neutron lifetime is negligibly short. Such an approximation is adequate if the reactivity is less than $\beta-2 \sqrt{\beta \lambda l}$ where $l=$ prompt neutron lifetime, and $\lambda=$ mean delayed neutron decay rate. This reduces the equation to

$$
(\beta-\rho) \frac{d n}{d t}=\left(\lambda \rho+\frac{d \rho}{d t}\right) n+l\left(\lambda S+\frac{d S}{d t}\right), \quad \beta-\rho \gg \sqrt{\beta \lambda l}
$$

where $S$ is the inhomogeneous source term. This equation may be solved by quadratures if $\rho(t)$ is given.

When the reactivity is a linear function of time, one can solve the equations without the neglect of the prompt lifetime. If we consider only one group of delayed neutrons the solution is (6).

$$
\begin{gathered}
n(t)=\alpha \int_{C}\left(1+\frac{z^{x}}{\lambda}\right)^{\beta \lambda / \gamma} \exp \left\{x\left(\rho_{0}+\gamma^{t}-\beta\right)-\frac{1}{2} \gamma l x^{2}\right\} d x \\
\rho(t)=\rho_{0}+\gamma^{t}
\end{gathered}
$$

and the contour $C$ in the complex z plane as well as the constant $\alpha$ are determined by the boundary conditions and the (constant) source strength s.

If the flux level is initially in equilibrium with the source the integral can be reduced to an integration along the real axis. Wallach has also extended this solution to the case of several delayed groups and obtains

$$
n(t)=\alpha \int_{0}^{\infty} \prod_{i}\left(1+\frac{\gamma x}{\lambda_{i}}\right)^{\beta_{i} \lambda_{i} / \gamma} \exp \left\{x\left(\rho_{0}+\gamma t-\beta\right)-\frac{1}{2} \gamma l x^{2}\right\} d x
$$

If the source term vanishes and if $\beta \lambda / \gamma$ is an integer an alternate form
of solution is

$$
\begin{gathered}
\gamma \geqslant 0 \quad n(t)=e^{-\lambda t} \frac{d^{p}}{d u^{p}} \int_{u_{1}}^{u} \exp \left\{\frac{\gamma}{2 l}\left(u^{2}-v^{2}\right)\right\} d v \quad p=\beta \lambda / \gamma \\
u=t+\frac{\rho_{0}-\beta+\lambda l}{\gamma}
\end{gathered}
$$




$$
\begin{array}{r}
\gamma<0 \quad n(t)=\exp \left(-\lambda t-\frac{|\gamma|}{2 l} u^{2}\right) \frac{d^{q-1}}{d u^{q-1}} \int_{u_{1}}^{u} \exp \left\{\frac{|\gamma|}{2 l}\left(u^{2}-v^{2}\right)\right\} d v \\
q=\beta \lambda /|\gamma|
\end{array}
$$

For non-integral values of $p$ (or $q$ ) it may often be adequate to interpolate the solution between nearest integral values.

Rodeback (2) has applied the Liouville approximation to the single delay group problem. The reactivity $\rho$ is assumed to be a given function of time but is independent of the neutron density $n$. Then with

$$
\omega=\frac{1}{2 l}(\rho-\beta-\lambda l) \quad \Omega^{2}=\omega^{2}+\frac{1}{l}\left[\lambda \rho+\frac{1}{2} \frac{\alpha \rho}{d t}\right]
$$

we obtain an approximate solution which is valid if $\Omega(t) \neq 0$

$$
n(t)=\frac{1}{\sqrt{\Omega}}\left\{A e^{\int^{t} p_{+} d t^{\prime}}+B e^{\int p_{-} d t^{\prime}}\right\} \quad p_{ \pm}=\omega \pm \Omega
$$

If $\Omega\left(t_{0}\right)=0$, one can use the WKB method ( $\left.\underline{8}\right)$ to pass across the "turning point." $\circ$ We find, in the usual manner

$$
n(t)=\sqrt{\frac{\psi}{\Omega}}\left[A I_{1 / 3}(\psi)+B I_{1 / 3}(\psi)\right] e^{\int^{t} \omega d t^{\prime}}
$$

where $\psi=\int_{t_{0}}^{t} \Omega\left(t^{\prime}\right) d t^{\prime}$

$\mathrm{Eq}(20.1)$ is useful if $\Omega(t)$ has only one zero. Otherwise the analysis must be extended by the standard methods. The accuracy of equation (20) or $(20.1)$ is determined by the behavior of the function $\rho(t)$; in general, reasonably accurate results are obtainable if $\left|l \frac{d f}{d t}\right| \ll \beta$.

The reactor kinetic equations may be rewritten from Equations (14.1), (14.2) in the form

$$
\begin{aligned}
& \frac{d n}{d t}=\frac{\beta}{l}\left[(r-1) n+\sum f_{i} w_{i}\right]+S \quad w_{i}=\frac{l \lambda_{i} c_{i}}{\beta_{i}} \quad f_{i}=\beta_{i} / \beta \\
& \frac{d \omega_{i}}{d t}=\lambda_{i}\left(n-\omega_{i}\right)
\end{aligned}
$$

The reactivity $r(n, \ldots ., t)$ is expressed in dollars $(r=\rho / \beta)$ and the ellipsis indicates that the reactivity may be a function of other variables as well. The defining equations of these other variables are assumed known.

An analytical side light might be mentioned here. In transient tests of reactors, attention has recently been paid to the reactivity of the reactor at the peak of the power excursion. Because the delayed neutrons 
are not in equilibrium during, a fast transient the reactivity is not zero when $d n / d t=0$. The quantity $r_{0}-r\left(t_{m}\right)$ (where $t_{m}$ is the time at which the maximum power is achieved) shows a minimum in the vicinity of $r_{0}=1$. When this effect was first observed at the SPERT reactor at the National Reactor Test Station, it was believed that this function could provide information on the mechanism of reactor shut down. It can, however, be shown (somewhat unfortunately) that the minimum is a result primarily of the delayed neutron lifetime and should therefore be independent of the reactor.

If we consider Eq (2l.1) with the source term set equal to zero (or negligibly small), we see that at the peak neutron density we have

$$
r_{0}-r\left(t_{m}\right)=r_{0}-1+\frac{1}{n} \sum_{i} f_{i} w_{i}
$$

If the reactor is on a slow period the delayed neutron precursors will be in equilibrium with the neutrons and the last two terms will almost cancel, (the reactivity of the system at the peak power point will be approximately zero). On the other hand, in a fast transient the delayed neutron precursors will contribute nothing and the inherent stability mechanism need compensate only for the reactivity in excess of prompt critical. The reactivity remaining in the system at the peak will therefore always be less than $\$ I$ and $i t$ will reach this value for reactivity inputs of only slightly greater than prompt critical, resulting in a rather sharp minimum at that point in the "compensated reactivity" curve. This minimum therefore cannot imply any complexity in the stabilizing mechanism other than that contained in the delayed neutrons themselves.

\section{NUMERICAI SOLUTION OF THE KINETICS EQUATIONS}

Several digital computer codes have been written in recent years to handle the reactor kinetics problem (9, 10, 11, 12). The calculation procedures used in these codes have usually been such as to contain severe limitations on the size of the time step as the calculation develops the fluxes at time $t+h$ from the fluxes at time $t$. The difficulties here have been outlined by Blue, et al (12). The basic problem lies in the existence of a solution to the linearized kinetics equations whose time dependence is given approximately by $\exp (-\beta t / l)$. The characteristic time $l / \beta$ can be very short (of the order of $10^{-2}$ seconds for thermal reactors and $10^{-5}$ seconds for fast reactors). On the other hand a reactor operating near critical is controlled by the delayed neutrons and the time scale is therefore of the order of seconds or even longer. One thus finds that it is necessary to calculate perhaps thousands of time points in order to obtain only a small change in reactor power. Except for an initial transient associated with a discontinuous change in reactivity, the flux is smoothly varying from point to point so that very little information is contained in these numbers. Such a calculation is therefore quite inefficient from the standpoint of computing time and furthermore, the great number of individual computations involved increases the effect of roundoff and other computational errors. 
The formal integration of equations (2l.1) is

$$
\begin{aligned}
& n(t+h)=n(t) e^{Q(0)}+\int_{0}^{h}\left\{\frac{\beta}{l} \sum_{i} f_{i} w_{i}(t+\xi)+S(t+\xi)\right\} e^{Q(\xi)} d \xi \\
& Q(\xi)=\frac{\beta}{l} \int_{t+\xi}^{t+h}\left[r\left(t^{\prime}\right)-1\right] d t^{\prime}
\end{aligned}
$$

There are difficulties with this form if it is used as the basis for calculation, these difficulties lie in the evaluation of the function $Q(\xi)$ and in the subsequent integration, if $r\left(t^{\prime}\right)$ is not constant.

H. Soodak (1I) has suggested a fruitful modification of this scheme;

$$
n(t+h)=n(t) e^{\alpha h}+\int_{0}^{h} R\left(n, w_{i}, \ldots, t+\xi\right) e^{\alpha(h-\xi)} d \xi
$$

where $\alpha=\frac{\beta}{l}[r(t)-1]$

and $R\left(n, w_{i}, \ldots, t+\xi\right)=\frac{\beta}{l}\left[\{r(t+\xi)-r(t)\} n(t+\xi)+\sum f_{i} \omega_{i}\right]+S$

so that $\alpha$ is a constant throughout the integration of a single time step. The equation in this form is still an exact expression. The numerical approximation arises in replacing the integral by a finite system.

The following scheme, which is related to the Runge-Kutta method and which can be considered a generalization of it, appears to give excellent results. It will be outlined for the case of a single dependent variable (first order equation). The extension to systems of linear equations follows in the same way as in the usual Runge-Kutta procedure (13).

$$
\frac{d n}{d t}=\alpha n+R(n, t)
$$

Define $C_{m}(x)=\int_{0}^{1} u^{m-1} e^{x(1-u)} d u \quad$ which are elementary functions, then

$$
\begin{aligned}
& n_{0}=n(t) \quad R_{0}=R\left(n_{0}, t\right) \\
& \delta_{1}=\frac{1}{2} h C_{1}\left(\frac{1}{2} \alpha h\right)\left[R_{0}+\alpha n_{0}\right]
\end{aligned}
$$




$$
\begin{array}{ll}
n_{1}=n_{0}+\delta_{1} & R_{1}=R\left(n_{1}, t+\frac{1}{2} h\right) \\
\delta_{2}=\frac{1}{2} h C_{2}\left(\frac{1}{2} \alpha h\right)\left[R_{1}-R_{0}\right] & \\
n_{2}=n_{1}+\delta_{2} & R_{2}=R\left(n_{2}, t+\frac{1}{2} h\right) \\
\delta_{3}=h C_{1}(\alpha h)\left[R_{0}+\alpha n_{0}\right]+2 h C_{2}(\alpha h)\left[R_{2}-R_{0}\right] & R_{3}=R\left(n_{3}, t+h\right) \\
n_{3}=n_{0}+\delta_{3} & R_{4}=R\left(n_{4}, t+h\right) \\
\delta_{4}=h\left\{2 C_{3}(\alpha h)-C_{2}(\alpha h)\right\} & {\left[R_{0}-2 R_{2}+R_{3}\right]} \\
n_{4}=n_{3}+\delta_{3} & {\left[R_{4}-R_{3}\right]} \\
\delta_{5}=h\left\{2 C_{3}(\alpha h)-C_{2}(\alpha h)\right\} & n(t)+\delta_{3}+\delta_{4}+\delta_{5}
\end{array}
$$

The functions $C_{m}(x)$ are given by

$$
C_{1}(x)=\frac{1}{x}\left[e^{x}-1\right] \quad C_{m+1}(x)=\frac{1}{x}\left[m C_{m}(x)-1\right]
$$

The basis of the algorithm may be outlined as follows:

The formal solution of Eq (23) is

$$
n(t+h)=n(t) e^{\alpha u}+\int_{0}^{u} R(n(\xi), t+\xi) e^{\alpha(u-\xi)} d \xi
$$

The first step computes $n(t+1 / 2 h)$ assuming $R(n, t+F)$ is a constant equal to Ro. This gives a first approximation for $n(t+1 / 2 h)$ from which an approximate value of $R(t+1 / 2 h)$ can be computed. We now assume that $R$ varies linearly over this interval to evaluate a better approximation to $n(t+1 / 2 h)$ and with this we improve our estimate of $R(t+1 / 2 h)$. With this value of $R$ we now use a linear variation in $R$ from $t$ to $t+h$ and obtain a first estimate of $n(t+h)$. This gives a first estimate of $R(t+h)$ which allows a quadratic variation of $R$, passing the parabola through the values $R(t), R_{2}$ and $R_{3}$. A second estimate for $n(t+h)$ and a second estimate for $R(t+h)$, follow, and from this the final value of $n(t+h)$ is calculated.

\section{ACCURACY ANALYSIS}

The difficulty which limits the usual calculation arises from the attempt to match, to some order, the Taylor expansion of the solution of the 
differential equation. In such a matching the exponential $e^{\alpha h}$ is replaced by a finite polynomial $1+\alpha h+\frac{1}{2} \alpha^{2} h^{2}+\ldots+\frac{1}{n !} \alpha^{n} h^{n}$. If $\alpha h$ is not small the polynomial is a poor approximation to the exponential. It is this which places the limit on the size, $h$, of the integration interval. In the present scheme, however, the exponential is retained intact and the Taylor expansion is made only on the slowly varying functions. The accuracy of the calculation is thus not limited by the magnitude of $\alpha h$ but instead the limitation comes from the Taylor expansion of the total solution.

As yet no complete analysis of the accuracy of this procedure has been made, but some special cases have been studied.

Equation soluble by quadratures - R not a function of $n$. If we have the equation

$$
\frac{d y}{d t}=\alpha y+R(t)
$$

the schome given above reduces to the modified Simpson"s Rule approximation:

$$
\begin{aligned}
y(t+h)= & y(t) e^{x}+h \int_{0}^{1} e^{x(1-u)}\left[(1-2 u)(1-u) R_{0}+4 u(1-u) R_{1 / 2}\right. \\
& \left.+u(2 u-1) R_{1}+\frac{1}{6} u\left(u-\frac{1}{2}\right)(u-1)\left\{h^{3} R^{\prime \prime \prime}+\frac{u}{4} h^{4} R^{i v}\right\}\right] d u
\end{aligned}
$$

where $x=\alpha h ; \quad R_{k}=R(t+k h)$

This expression as it stands is exact if the third and fourth derivatives $R^{\prime \prime \prime}$ and $R^{2}$ are evaluated at appropriate points in the interval $(t, t+h)$. If $R_{1 / 2}$ and $R_{1}$ are calculated accurately the error in using the quadratic fit Is given by

$$
\begin{array}{cc}
\varepsilon=\frac{h^{5}}{720}\left[K_{3} \alpha R^{\prime \prime \prime}-\frac{1}{4} K_{4} R^{i v}\right] & \\
K_{3}(x)=30\left[C_{3}(x)-2 C_{4}(x)+C_{5}(x)\right] & K_{3}(x) \rightarrow 1 \\
K_{4}(x)=60\left[-C_{3}(x)+3 C_{4}(x)-2 C_{5}(x)\right] & K_{4}(x) \rightarrow 1
\end{array}
$$

The case of particular interest is $\alpha<0,|\alpha h| \gg 1$,

then $K_{3} \approx 15(\alpha h)^{-3} \quad K_{4} \approx 60(\alpha h)^{-2}$

and the error is

$$
\varepsilon \approx \frac{1}{48} h^{4}(\alpha h)^{-2} R^{\prime \prime \prime} \quad(\alpha h)^{2} \gg 1 .
$$


An error analysis of this system for $R$ dependent on both $t$ and $n$ is involved, since the number of algebraic manipulations required if all terms of order $\mathrm{h}^{4}$ are retained becomes oppressive. In the case $\alpha=0$ however, the formulas reduce to a Runge-Kutta system in which the error can be shown to be at least of order $h^{4}$. The system, however, is not an optimum set since an accuracy of this order can be obtained with existing Runge-Kutta procedures using only four (rather than five) intermediate calculations.

\section{APPLICATIONS TO SPECIFIC REACTOR SYSTEMS}

A. KEWB REACTOR (HOMOGENEOUS SOLUTION)

A homogeneous reactor of the water boiler type (14) is inherently stable under a wide range of reactivity excursions. The analysis of its kinetic behavior leads to a description of this reactivity compensation in terms of the temperature increase in the core and the production of radiolytic gas (decomposition of water). The radiolytic gas does not appear instantaneously as gas bubbles in the solution but only after a time lapse required for bubble formation. The mathematical description of the reactor is given by Eq $(21.1)$ and (2l.2) augmented by the following equations for the reactivity as a function of the state of the reactor (14)

$$
\begin{aligned}
& r(t)=r_{0}(t)+\alpha T+\phi V \\
& \frac{d T}{d t}=K\left(n-n_{0}\right) \\
& \frac{1}{\nu} \frac{d^{2} V}{d t^{2}}+\left(1+\frac{\sigma}{\nu}\right) \frac{d V}{d t}+\sigma V=G\left(n-n_{0}\right)
\end{aligned}
$$

where $\frac{1}{\nu}=$ time delay for bubble formation

$$
\begin{aligned}
& \frac{1}{\sigma}=\text { residence time of bubbles in reactor core } \\
& G=\text { rate of radiolytic decomposition of water }=4 \mathrm{~cm}^{3} \mathrm{kw}^{-1} \mathrm{sec}^{-1} \\
& K=\text { reciprocal heat capacity in appropriate units } \\
& \alpha=\text { temperature coefficient of reactivity }=-0.02 \$ /{ }^{\circ} \mathrm{C} \\
& \phi=\text { bubble volume coefficient of reactivity }=-0.005 \$ \mathrm{~cm}^{-3}
\end{aligned}
$$

The experimental results have not yet been completely correlated by this model, but qualitative agreement has been achieved.

\section{B. SPERT REACTOR - A HETEROGENEOUS SYSTEM}

The analysis of the SPERT transients (15) is based on the assumption that the reactivity can be defined in terms of the mean moderator density and that a decrease in the number of hydrogen atoms per unit volume in the 
core (either as the result of thermal expansion or as the result of gas bubble formation resulting from boiling) will decrease the reactivity. The reactivity is then

$$
r=r_{0}-a y(t)
$$

The increase in the temperature of the fuel plates, above the initial (equilibrium) value, $\theta(t)$, is represented by a first order equation

$$
\frac{d \theta}{d t}+b \theta=\frac{1}{h} n
$$

The laws determining the growth of bubbles in the moderator is unknown under the transient condition present in the reactor but a reasonable assumption is

$$
\frac{d v_{b}}{d t}+c_{2} v_{b}=c_{1} \theta
$$

Expansion of the water and of the fuel plates (thus forcing water out of the core) can be combined into a term $v_{w}$ dependent to varying extent on both the fuel plate temperature and the power level (or neutron density)

$$
\frac{d v_{w}}{d t}=s_{1} n+s_{2} \theta
$$

and $y(t)=v_{b}(t)+v_{w}(t)$

Horning (16) has analyzed this system assuming that the neutron density can be expressed as a series of exponentials

$$
n(t)=n_{0} e^{p t}+n_{1} e^{2 p t}+n_{2} e^{3 p t}+\ldots
$$

This form leads to a specific expression for the reactivity compensated at the time of peak power

$$
r_{0}-r\left(t_{m}\right)=p\left[\frac{\ell}{\beta}+\sum_{i} \frac{f_{i} \lambda_{i}}{\left(\lambda_{i}+p\right)\left(\lambda_{i}+2 p\right)}\right]
$$

where $p$ is the dominant period of the inhour equation

$$
r_{0}=p\left[\frac{l}{\beta}+\sum_{i} \frac{f_{i}}{\lambda_{i}+p}\right] \text {. }
$$

For small oscillations of the power the linearization of equations (2l), (29), (30), (31) yield an oscillatory solution with a frequency $\omega$ which is given approximately by the relation

$$
\omega^{2}=a c, n / h
$$

where $\mathrm{n}$ is the equilibrium neutron level. Equation (35) applies to stable oscillations at low power. There is however a critical power level above which the oscillations become divergent 


$$
n>\frac{h}{a c_{1}}\left[\frac{\beta}{l}\left\{c_{2}+b-\sum_{i} f_{i} \lambda_{i}\right\}+c_{2}^{2}\right]
$$

Spontaneous power oscillations have been observed in the SPERT reactor and it has been possible to correlate this behavior qualitatively with two time delays in the reactor system. The first in the time constant, $h / \rho L c_{1}$, which represents the delay in the production of steam bubbles. (The quantity $\rho I$ is the latent heat of vaporization of water per $\mathrm{cm}^{3}$ of steam and $h$ is in terms of reactor power rather than neutron density.) The second is the time constant $1 / c_{2}$ which represents the residence time of steam bubbles in the reactor core. It has been possible to choose these two delays to account for the principal features of the instability: the dependency of frequency on mean reactor power, the relation between peak power and the width of the power peak, and the relation between the maximum and minimum of the power oscillation. The model represented by Eqs (29) and (32) will be confirmed if out-of-pile measurements of the above two delay times yield the same values as those required ad hoc for the SPERT transient data. 


\section{REFERENCES}

(1) Ussachoff, L. N., Proceedings of the International Conference on Peaceful Uses of Atomic Energy, Vol. 5, p.503 (P/656)

(2) Henry, A. F., Nuclear Science and Engineering: 3,52(1958); Henry, A. F。 and Curlee, N. J., Nuclear Science and Engineering, (to appear)

(3) Welton, T. A., "Kinetics of Stationary Reactor Systems," Peaceful Uses of Atomic Energy vol. 5. p.377 (1956) (P/610)

(4) Goertzel, G., Reactor Handbook, Chap. 1.6, Reactor Dynamics, USAEC (1955) (AECD-3645)

(5) Soodak, H., American Nuclear Society, 2nd Winter Meeting, Abstract 5-1. October 28, 1957; Skinner, R。 E., and Cohen, E。 R。, American Nuclear Society, 2nd Winter Meeting, Abstract 5-2. October 28, 1957.

(6) Wallach, S., WAPD-13, (unpublished)

(7) Rodeback, G. W., NAA-SR-1854 (1957), unpublished

(8) Morse, P. M., and Feshback, H., Methods of Theoretical Physics, McGrawHill Book Co。, New York (1953), p. 1092.

(9) Sangren, W. C., Kinetics Calculations for Homogeneous Reactors, ORNIml205 (1955), unpublished

(10) Brown, H. D., Nucl. Science and Engineering, 2:687 (1957); Ash, M., Journ. Applied Physics 27:1030 (1956)

(11) Wills, I., Krumbein, A., Soodak, H., Certaine, J., Gruber, W., Amer. Nuclear Soc. Meeting, Oct. 28, 1957 (Abstract 5-3)

(12) Blue, E., Blue, L。 R., Cohen, E。 R。, Dennemyer, R。F。, Hetrick, D. L。, Schwartz, A., Amer. Nuclear Society Meeting, Oct. 28, 1957 (Abstract 5-4)

(13) Milne, W. E., Numerical Solution of Differential Equations, John Wiley and Sons, Inc., New York (1953), p-72; Hildebrand, F。 B., Introduction to Numerical Analysis, McGraw-Hill, New York (1956), p. 233

(14) Remley, M. Eo, et al. "Experimental Studies on the Kinetic Behavior of Water Boiler Type Reactors, Geneva Conference (1958)

(15) Schroeder, F., Forbes, S. G., Nyer, W. E., Bentzen, F. L., and Bright, G. O., Nuclear Science and Engineering, 2:96 (1957)

(16) Horning, W. A. and Corben, H. C., "Theory of Power Transients in the SPERT-I Reactor," Ramo-Wooldridge Corp., ERI-109 (unpublished) 